\title{
BMJ Open Protocol to describe the analysis of text-based communication in medical records for patients discharged from intensive care to hospital ward
}

\author{
Jeanna Parsons Leigh, ${ }^{1,2}$ Kyla Brown, ${ }^{3}$ Denise Buchner, ${ }^{4}$ Henry T Stelfox ${ }^{1,2,4}$
}

To cite: Parsons Leigh J, Brown K, Buchner D, et al. Protocol to describe the analysis of text-based communication in medical records for patients discharged from intensive care to hospital ward. BMJ Open 2016;6:e012200. doi:10.1136/bmjopen-2016012200

- Prepublication history and additional material is available. To view please visit the journal (http://dx.doi.org/ 10.1136/bmjopen-2016012200).

Received 8 April 2016 Revised 7 June 2016 Accepted 14 June 2016

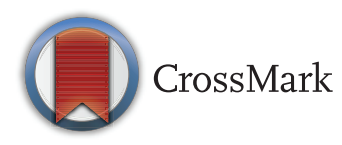

\footnotetext{
${ }^{1}$ Department of Critical Care Medicine, University of Calgary, Calgary, Alberta, Canada

${ }^{2}$ Alberta Health Services, Calgary, Alberta, Canada ${ }^{3}$ W21C Research and Innovation Centre, Institute of Public Health, University of Calgary, Calgary, Alberta, Canada

${ }^{4}$ Department of Community Health Sciences, University of Calgary, Calgary, Alberta, Canada
}

Correspondence to Dr Henry T Stelfox; tstelfox@ucalgary.ca

\begin{abstract}
Introduction: Effective communication during hospital transitions of patient care is fundamental to ensuring patient safety and continuity of quality care. This study will describe text-based communication included in patient medical records before, during and after patient transfer from the intensive care unit (ICU) to a hospital ward ( $\mathrm{n}=10$ days) by documenting (1) the structure and focus of physician progress notes within and between medical specialties, (2) the organisation of subjective and objective information, including the location and accessibility of patient data and whether/ how this changes during the hospital stay and (3) missing, illegible and erroneous information.
\end{abstract}

Methods: This study is part of a larger mixed methods prospective observational study of ICU to hospital ward transfer practices in 10 ICUs across Canada. Medical records will be collected and photocopied for each consenting patient for a period of up to 10 consecutive days, including the final 2 days in the ICU, the day of transfer and the first 7 days on the ward ( $\mathrm{n}=10$ days). Textual analysis of medical record data will be completed by 2 independent reviewers to describe communication between stakeholders involved in ICU transfer.

Ethics and dissemination: Research ethics board approval has been obtained at all study sites, including the coordinating study centre (which covers 4 Calgarybased sites; UofC REB 13-0021) and 6 additional study sites (UofA Pro00050646; UBC PHC Hi4-01667;

Sunnybrook 336-2014; QCH 20140345-01H;

Sherbrooke 14-172; Laval 2015-2171). Findings from this study will inform the development of an evidencebased tool that will be used to systematically analyse the series of notes in a patient's medical record.

\section{INTRODUCTION}

High-quality communication between healthcare providers is essential for effective coordination of patient care from hospital admission through to hospital discharge. ${ }^{1}$ However, patterns of communication between multiprofessional providers are often complex and
Strengths and limitations of this study

- Data will be collected from 10 sites (7 cities, four provinces) across Canada.

- Data analysis will inform the development of a tool that has been designed with the intent to improve communication in the ICU to hospital ward transfer process.

- Strong potential to improve the continuity of care plans.

- Applicability to electronic medical records will not be evaluated.

- Results may not be generalisable to hospitals in different health jurisdictions.

transpire through various forms including face to face, textual (paper based and electronic) and over the phone communication. ${ }^{2}$ The risk of communication failures is high, with one in two patients experiencing a breakdown in communication during their hospital length of stay. ${ }^{3}{ }^{4}$ Such breakdowns are associated with reduced quality of patient care, increased preventable medical errors ${ }^{3}$ and adverse events, ${ }^{4}$ and patient and family members who are dissatisfied with the quality of care. ${ }^{5}$

This study will describe and evaluate textbased communication during patient transfer from ICU to hospital ward. Specifically, we will analyse clinical notes and transfer tools documented in patient medical records for up to 10 calendar days, including 2 days before ICU to hospital ward transfer, the day of transfer and up to 7 days after the transfer depending on patient length of hospital stay. For the purposes of this study, we define 'medical record' as the record of a patient's medical information (eg, history, care or treatments received, test results, diagnoses, and medications taken, admission and discharge summaries, etc). To ensure the feasibility of the study, we have restricted our focus to physician notes (admission, progress, 
consultation and discharge). The goal of this study was to describe the content and structure of text-based patient medical records during patient transfer from ICU to hospital ward. Results will shed light on the nature of text-based communication between providers during ICU to hospital ward transitions and what might be done to improve it.

\section{Communication during hospital transitions of care}

Hospital transitions of care (ie, patients moving from one provider to another or one care location to another) are periods in healthcare delivery that are particularly vulnerable to breakdowns in communication. In particular, the transfer of patients between healthcare teams can lead to the transmission of fragmented information and low-quality patient care. ${ }^{6}$ Patient transfer from the ICU to hospital ward is a challenging and highrisk transition of care as the sickest patients with the most complex needs move from a high-intensity care environment to an environment ${ }^{7}$ with far fewer resources. During this transition, patient information must undergo a multiprofessional (ie, nurse to nurse, physician to physician) and multispecialty (ie, ICU provider to ward provider) transition. Additionally, differences between ICU and hospital ward settings and differences in clinical focus between professions and specialties $^{8-10}$ are linked to poor communication that causes confusion about care plans ${ }^{11-13}$ and patient conditions, ${ }^{14}$ decisions that are based on inaccurate or incomplete information, ${ }^{15}$ redundant testing and treatments ${ }^{16}$ and medication errors ${ }^{3}$ in the ICU to ward transfer process.

\section{Text-based communication}

A patient's medical record performs a vital function in maintaining continuity of care across the healthcare continuum because it is the central mechanism within which textual communication between providers is recorded. ${ }^{17}$ In addition, a patient can experience numerous instances of provider handover during their hospital length of stay, but the documentation included in the medical record stays with the patient as an objectified representation of their history (eg, medications prescribed), daily goals of care and treatment plan. ${ }^{18}$ When patients are unable to speak or advocate for themselves —as is common with many ICU patients-the medical record is even more crucial because it stands in for the 'patient's story'. ${ }^{19}{ }^{20}$ While the significance of effective provider communication to patient safety and the provision of quality care have been well documented, ${ }^{5} 2122$ less is known about the effectiveness of textual communication in coordinating high-quality care during patient transitions from the ICU to a hospital ward, ${ }^{8}$ and few tools exist to evaluate this. ${ }^{23}$

\section{Physician progress notes}

Medical records are an important source of patient information and often contain handwritten or typed physician progress notes and document provider assessments, investigations, current problem lists and management strategies. ${ }^{24}$ Consistency in the structure and style of progress notes included in the medical record is associated with ease and efficiency of provider review, including the timely identification of key facts that are pivotal to patient care and planning processes. ${ }^{25}$ However, anecdotal evidence suggests that physicians with different clinical backgrounds (ie, specialties) often take differing approaches to medical record documentation, ${ }^{8}$ leading to breakdowns in communications, discrepancies in patient care, treatment errors, conflicting decisionmaking and a lower quality of care. ${ }^{26-28}$ There is a dearth of information about how physicians with different clinical backgrounds provide written documentation in patient medical records. To the best of our knowledge, no tools are currently available to measure these particular discrepancies.

\section{Standardisation in clinical note taking}

Efforts to standardise clinical note taking have been made to improve consistency and communication. ${ }^{25}$ In particular, widely accepted criteria for clinical progress note entries is that the note should contain the patient's name, hospital identification number, date, time and also the name and designation of the physician who is entering the notation. ${ }^{29}$ In addition, entries should be presented in chronological order to reflect a continuum of patient care and entries should occur immediately following an event. ${ }^{29} \mathrm{~A}$ prominent strategy for progress note documentation follows the subjective, objective, assessment and plan (SOAP) structure. ${ }^{30} 31$ This structure advocates for a problem-oriented medical record $(\mathrm{POMR})^{30}$ and has been implemented in a range of hospital settings. ${ }^{31}{ }^{32}$ It has been demonstrated that the use of the SOAP structure leads to more consistent and reliable documentation of physician progress notes ${ }^{30}$ which can enhance communication between providers ${ }^{28} 32$ and bolster trainee education initiatives. ${ }^{33}$ However, the SOAP format has been criticised for failing to take the experiences and observations of patients and families into account. ${ }^{34}$ Furthermore, comorbidities and complications are poorly documented when the SOAP structure is followed without a template to prompt their input. $^{35}$

We propose a descriptive textual analysis study to describe text-based communication included in patient medical records before, during and after patient transfer from the ICU to medical ward. We will collect and analyse information that describes the content and structure of physician progress notes within and between medical specialties, the organisation of subjective and objective information, as well as missing, illegible and erroneous information included in the medical record. Data analysis will identify opportunities to improve communication during the ICU to hospital ward transfer process and support the development of an ICU transfer toolkit. 


\section{OBJECTIVES}

\section{Overarching objective}

To describe communication content and structure during transfer from ICU to hospital ward as depicted in patients' medical records.

\section{Specific objectives}

1. Establish chart organisation and identify similarities and differences in charting across specialties and units.

2. Describe how the medical record is used by providers (ie, how medication information is stored, how often previous notes are referred to, type of progress note written, etc) in the ICU to ward transfer process to improve overall usability and effectiveness of the medical record.

3. Determine where breaks in communication and/or the loss of information occur in the medical record and the impact this has on patient care following transfer from the ICU to hospital ward.

\section{METHODS/DESIGN}

\section{Study design}

We propose to conduct a textual analysis of patient medical records that have been collected as part of the broader prospective cohort study, 'Developing an Evidence-Informed Intensive Care Unit Discharge Tool'. ${ }^{36}$ Specifically, physician progress and consultation notes, transfer tools, admission and discharge summaries included in the patient's medical record around the time of ICU to ward transfer-2 days prior to ICU transfer, the day of transfer and up to 7 days post-transfer to the accepting hospital ward (depending on length of hospital stay), totalling up to 10 consecutive calendar days-will be analysed. We anticipate that the 10-day time period proposed will capture the majority of textbased communication related to the transition of patient care from the ICU to hospital ward.

Study sites for data collection

- St Paul's Hospital, Vancouver, British Columbia

- University of Alberta Hospital, Edmonton, Alberta

- Foothills Hospital, Calgary, Alberta

- Rockyview General Hospital, Calgary, Alberta

- Peter Lougheed Centre, Calgary, Alberta

- South Calgary Health Campus, Calgary, Alberta

- Queensway Carleton Hospital, Ottawa Ontario

- Sunnybrook Health Sciences Centre, Toronto, Ontario

- CHU de Quebec (Hôpital de l'Enfant-Jésus), Quebec City, Quebec

- Centre Hospitalier Universitaire de Sherbrooke, Sherbrooke, Quebec

\section{Study population}

Patients will be eligible to participate in the study if they are admitted to a study ICU and transferred to a hospital ward within the same facility. Eligible participants must be 18 years or older, admitted to a medical/surgical ICU and be able to consent or have surrogate consent. Eligible participants must also be able to speak English or French.

\section{Consent}

Patients whose medical records are included in this study provided informed consent to have their medical records copied, de-identified and reviewed as part of the larger ICU discharge study. Informed consent was provided by patients who were determined to have the capacity to consent through a modified Aid to Capacity Evaluation tool. ${ }^{37}$ Patients who did not have capacity to consent were included in the study if consent was provided by a surrogate decision-maker.

\section{Data}

Each study site across Canada proposes to enrol 50 consecutive patients $(n=500)$ who are eligible to participate in the study and that satisfy the inclusion criteria and consent to participate. ${ }^{37}$ Patient medical records will be photocopied, de-identified, assigned a unique identifier and imported into NVivo V.10 for analysis. The contents of the medical record are anticipated to contain (1) physician progress note (whether in a dedicated physician progress note section or multidisciplinary section), (2) consultation notes, (3) admission and/or discharge summaries, (4) ICU patient transfer checklist or other transfer tools and (5) orders if part of a transfer tool.

\section{ANALYTICAL PLAN \\ Overview}

Analysis of de-identified patient medical records will be conducted in three phases (figure 1). In phase I, two investigators with expertise in medical record review will review a subsection of the data while employing an 'open coding' methodology ${ }^{38}$ where they will begin to define and develop themes of interest and experiment with labelling concepts. The unit of analysis will be each individual note clustered in the patient's larger medical record. Study objectives (ie, to identify similarities and differences in charting across specialties and units, to determine where breaks in communication and/or the loss of information is occurring, and to investigate how the medical record is used by providers) will be the anchoring principles that guide this initial stage of work.

Drawing on their independent preliminary analysis and observations, investigators will work collaboratively to reach agreement on pertinent themes and concepts emerging out of the data. Shared findings will then be presented to a subset of the larger research team (including one ICU physician, one ward physician and three health researchers) for feedback and discussion and the development of a shared coding framework.

Phase II of analysis will involve sharing this preliminary coding framework with ICU and ward stakeholders to gain a broader perspective on the framework 

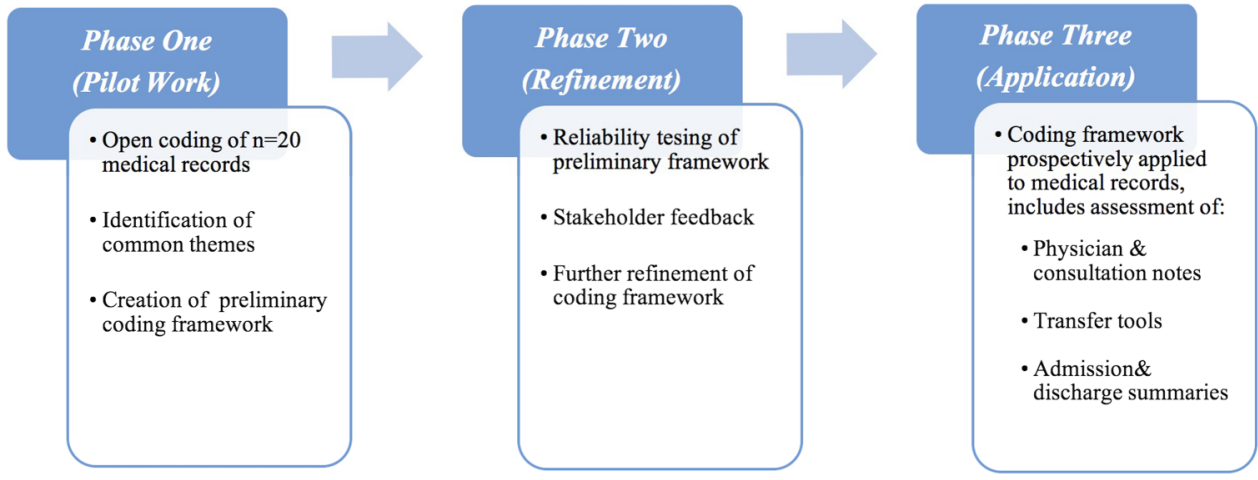

Figure 1 Summary of methodological approach.

developed by the research team. Following this exercise and the implementation of suggested revisions, two investigators will test the updated framework for interrater and intrarater reliability by analysing a sample $(n=10)$ of the medical records. Results of this testing will inform the final version of the coding framework. In phase III, this framework will be prospectively applied to the 500 patient medical records. A summary of this approach is depicted below.

\section{Phase I}

A pilot project was completed in November 2015 with the purpose of pretesting our chosen methodological approach on a subset of patient medical records. Medical records collected from one study site $(n=20)$ were reviewed and coded by two investigators (JPL, KB). As described above, investigators employed an opencoding methodology by independently documenting patterns, themes and categories of analysis emerging out of the data. This was an exploratory phase of work with the goal of orienting the researchers to the ways that patient information, problem lists and events are documented in the medical record. After individually analysing each of the 20 medical records, the investigators came together to compare notes and findings. Common themes and features were then noted across cases (ie, medical records) first verbally and then via the construction of detailed memos. ${ }^{39}$ Four main themes were independently identified by investigators as being in need of further exploration: organisation (location and order of information), readability (legibility and comprehension), focus (how emphasis is placed) and structure (style and design). Below is a synopsis of these categories, including related questions to be considered during codebook development (box 1).

Presentation of preliminary findings to the advisory group to the larger study led to the creation of two sets of codes, simple and complex, to guide the development of a refined coding framework. Simple codes denote instances in a patient medical record that capture descriptive statistics and counts of information, such as if the note was signed, properly dated and time stamped. Complex codes denote codes that capture multifaceted issues that transcend variations in focus and style in an individual unit of analysis (eg, inconsistencies in the problem list over time, contradictory accounts or information, etc) and will be used to describe and compare text-based communication within patient medical records (with detailed memos being used to supplement the codes). Below is a synopsis of components that make up this preliminary coding framework (figure 2) which has been developed from the themes identified in phase I and supplemented by background work for this project

\section{Box 1 Emerging themes from medical record analysis}

Emerging themes of interest

Organisation

- Is the medical record organisation standard? Is the location where information is recorded within a medical record consistent?

- How is the medical record maintained and managed? To what extent is the organisation and completeness of the medical record maintained?

- What is the interplay between electronic and paper-based charts?

Readability

- Is the medical record legible? To what extent can users of the medical record extract relevant information?

- How frequently are abbreviations used within and between specialties? Do abbreviations potentially impair communication of information?

Focus

- Is there variation in focus between providers within a specialty and between providers of different specialties?

- Is there variation in how a specific type of content (eg, relating to illness, treatment, medication, patient history, etc) is documented?

- What is the purpose of a note? Memory aid, documentation, communication, etc.

Structure

- Is there variation in the structure of physician progress notes between providers within a specialty and between providers of different specialties? Does this matter and if so how?

- How is the note written (point form, sentences or single words)?

- Is there evidence that previous notes are reviewed?

- To what extent are incorrect facts perpetuated in the record? 


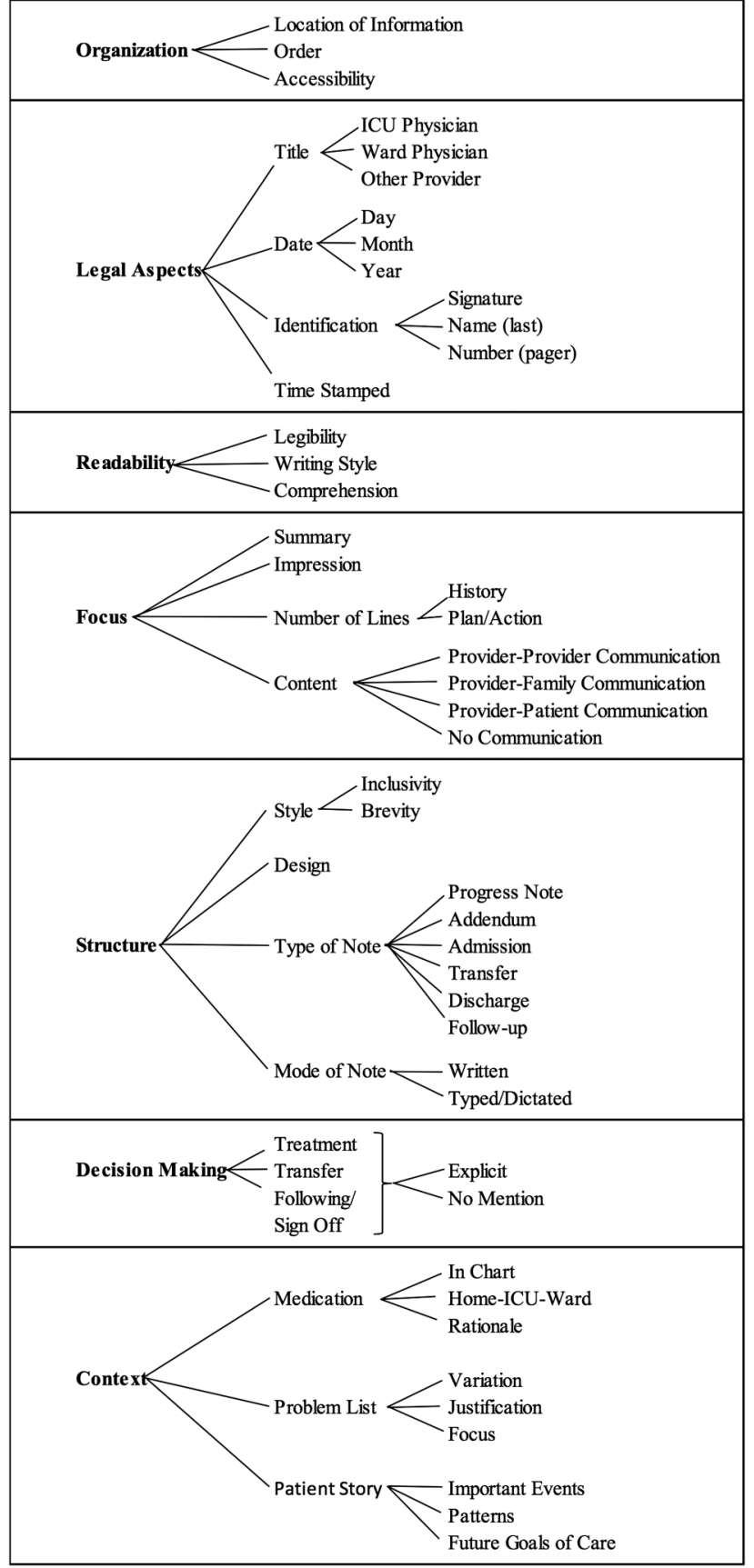

Figure 2 DRAFT (the proposed components are draft and likely to evolve as analysis unfolds) central components of medical record analysis.

(eg, literature review) and the expertise and experience of the larger research team. A more comprehensive version of the framework including a detailed list of proposed codes with examples of when and how to apply is also provided (see online supplementary appendix A).

\section{Phase II}

In phase II of this project, the preliminary coding framework will be shared with ICU and hospital ward stakeholders (eg, a sample of front-line providers and decision-makers responsible for patient care) to gain a broader perspective on the codes identified and developed and to ensure that the coding framework sufficiently captures all important elements of text-based communication in the ICU transfer process. Two investigators will then use the refined coding framework to analyse a small portion of the medical records $(n=10)$ to test for inter-rater and intrarater reliability. ${ }^{40}$

\section{Phase III}

Based on stakeholder feedback and reliability testing, the coding framework will be further refined and then prospectively applied to the remaining patient medical records $(n=470) .{ }^{i}$ Specifically, reviewers will assess each medical record in chronological order, including ICU admission note, physician and consultation notes (2 days prior to ICU discharge, day of discharge and 7 days postdischarge), text-based transfer tools (within and across specialties), ICU transfer summary, ward admission notes and hospital discharge note (as available). Each note included in the medical record will be coded line by line during the 10-day time frame. Reviewers will then draw on the coded data to develop a summary report that describes written communication in the patient's medical record during the time of transfer from ICU to hospital ward.

\section{RELEVANCE OF FINDINGS}

Streamlining and standardising communication during hospital transitions of care plays a vital role in reengineering ICU discharge and bridging vulnerable moments in healthcare delivery. It is expected that this work will make a significant contribution to the improvement of patient care by: (1) providing a comprehensive description of the textually documented structure and processes of ICU discharge; (2) adding to a conceptual framework of textual communication and (3) generating an evidence informed tool that will streamline ${ }^{40}$ communications and enhance patient care. Furthermore, lessons learnt from this work will likely be applicable to other transitions of care that involve written communication (eg, transitions of care between providers within a healthcare setting, patient discharge from hospital) and future research could extend this work to include a focus on interdisciplinary documentation (eg, nursing notes).

\section{NEXT STEPS}

This study has the potential to improve the care of critically ill patients during hospital transitions of care by providing a comprehensive description of text-based communication during the ICU to hospital ward transfer process in 10 hospitals across Canada. This will include

${ }^{\mathrm{i}}$ The remaining number of patient medical records is 470 as 20 were initially reviewed to identify common themes, and 10 were used for inter-rater/intrarater reliability tests. 
an analysis of physician progress notes, dictated admission, transfer and discharge summaries and text-based transfer tools. Next steps include further developing and refining our coding framework based on a process of stakeholder feedback and reliability testing, and prospectively applying the updated framework to patient medical data.

\section{DISSEMINATION}

Data collected from this study will inform the development of a communication tool that will be rolled out as part of a customised, multicomponent ICU discharge tool kit for ICU to hospital ward transfers. ${ }^{36}$ The overarching goal of this tool kit will be to improve patient outcomes and care across the healthcare continuum by streamlining communication and targeting behaviour change for our stakeholders (patients, families, providers, managers). Key deliverables developed from this study will be made available in English and French. We anticipate that no ethical or safety considerations will arise from this research.

\section{Twitter Follow Jeanna Parsons Leigh at @JParsonsLeigh}

Contributors All authors contributed to the conception (HTS) or design (JPL, $\mathrm{KB}, \mathrm{DB}$ ) of the work; and drafting (JPL, KB) or revising (DB, HTS) the work for important intellectual content; and provide final approval of the manuscript and agree to be accountable for the accuracy and integrity of the work.

Funding This work was supported by Technology Evaluation in the Elderly Network (TVN) (CORE 2013-12A).

Competing interests None declared.

Ethics approval Research ethics board approval has been obtained at all study sites including the coordinating study centre (which covers 4 Calgary based sites) (UofC REB 13-0021) and 6 additional study sites (UofA Pro00050646; UBC PHC Hi4-01667; Sunnybrook 336-2014; QCH 20140345-01H; Sherbrooke 14-172; Laval 2015-2171).

Provenance and peer review Not commissioned; externally peer reviewed.

Data sharing statement Additional unpublished data from this study are available to research team members as outlined in our ethics certificate (UofC REB 13-0021).

Open Access This is an Open Access article distributed in accordance with the Creative Commons Attribution Non Commercial (CC BY-NC 4.0) license, which permits others to distribute, remix, adapt, build upon this work noncommercially, and license their derivative works on different terms, provided the original work is properly cited and the use is non-commercial. See: http:// creativecommons.org/licenses/by-nc/4.0/

\section{REFERENCES}

1. Dalal A, Schnipper J. Care team identification in the electronic health record: a critical first step for patient-centered communication. J Hosp Med 2016;00:1-5.

2. Benham-Hutchins MM, Effken JA. Multi-professional patterns and methods of communication during patient handoffs. Int J Med Inform 2010;79:252-67.

3. Bell CM, Brener SS, Gunraj N, et al. Association of ICU or hospital admission with unintentional discontinuation of medications for chronic diseases. JAMA 2011;306:840-7.

4. Camiré E, Moyen E, Stelfox HT. Medication errors in critical care: risk factors, prevention and disclosure. CMAJ 2009;180:936-41.

5. Li P, Stelfox HT, Ghali WA. A prospective observational study of physician handoff for intensive-care-unit-to-ward patient transfers. Am J Med 2011;124:860-7.
6. Rochester-Eyeguokan CD, Pincus KJ, Patel RS, et al. The current landscape of transitions of care practice models: a scoping review. Pharmacotherapy 2016;36:117-33.

7. Dutton RP, Cooper $\mathrm{C}$, Jones A, et al. Daily multidisciplinary rounds shorten length of stay for trauma patients. J Trauma 2003;55:913-19.

8. Riesenberg LA, Leitzsch J, Massucci JL, et al. Residents' and attending physicians' handoffs: a systematic review of the literature. Acad Med 2009;84:1775-87.

9. Apker J, Mallak L, Gibson S. Communicating in the "gray zone": perceptions about emergency physician-hospitalist handoffs and patient safety. Acad Emerg Med 2008;14:884-94.

10. Pronovost $P$, Vohr E. Safe patients, smart hospitals: how one doctor's checklist can help us change health care from the inside out. New York, NY: Hudson Street Press, 2010.

11. Sutcliffe KM, Lewton E, Rosenthal MM. Communication failures: an insidious contributor to medical mishaps. Acad Med 2004;79:186-94.

12. Cook R, Render M, Woods D. Gaps in the continuity of care and progress on patient safety. BMJ 2000;320:791-4.

13. Ye K, McD Taylor D, Knott JC, et al. Handover in the emergency department: deficiencies and adverse effects. Emerg Med Australas 2007;19:433-41.

14. Arora V, Johnson J, Lovinger D, et al. Communication failures in patient sign-out and suggestions for improvement: a critical incident analysis. Qual Saf Health Care 2005;14:401-7.

15. Joint Commission on Accreditation of Healthcare Organizations, USA. Using medication reconciliation to prevent errors 2006. http:// www.jointcommission.org/sentinel event alert issue 35 using medication_reconciliation_to_prevent_errors/ (accessed 2 Feb 2016).

16. Niès J, Colombet I, Zapletal E, et al. Effects of automated alerts on unnecessarily repeated serology tests in a cardiovascular surgery department: a time series analysis. BMC Health Serv Res 2010;10:70.

17. May-Miller H, Hayter J, Loewenthal L, et al. Improving the quality of discharge summaries: implementing updated Academy of Medical Royal Colleges standards at a district general hospital. BMJ Qual Improv Rep 2015;4:pii: u207268.w2918.

18. Rankin J, Campbell M. Institutional Ethnography (IE), nursing work and hospital reform: IE's cautionary analysis. Forum Qual Soc Res 2009;10:Art. 8.

19. Rankin JM. The rhetoric of patient and family centered care: an institutional ethnography into what actually happens. J Adv Nurs 2015;71:526-34.

20. Varpio L, Rashotte J, Day I, et al. The EHR and building the patient's story: a qualitative investigation of how EHR use obstructs a vital clinical activity. Int J Med Inform 2015;84:1019-28.

21. National Transitions of Care Coalition. Improving transitions of care. NTOCC 2008:1-46. http://www.ntocc.org/Portals/0/PDF/Resources/ PolicyPaper.pdf

22. Johnson C, Carta T, Throndson K. Communicate with me: information exchanges between nurses. Can Nurse 2015;3:24-7.

23. Stelfox HT, Lane D, Boyd JM, et al. A scoping review of patient discharge from intensive care: opportunities and tools to improve care. Chest 2015;147:317-27.

24. Wilcox L, Lu J, Lai J, et al. eds. Physician-driven management of patient progress notes in an intensive care unit. 28th International Conferences on Human Factors in Computing Systems. April 10-15 2010. New York, NY, USA; 2010.

25. Pollard SE, Neri PM, Wilcox AR, et al. How physicians document outpatient visit notes in an electronic health record. Int J Med Inform 2013;82:39-46.

26. Sleszynski S, Glonek T, Kuchera W. Outpatient osteopathic single organ system musculoskeletal exam form series: validation of the outpatient osteopathic SOS musculoskeletal exam form, a new standardized medical record. J Am Osteopath Assoc 2004;104:423-38

27. Embi PJ, Yackel TR, Logan JR, et al. Impacts of computerized physician documentation in a teaching hospital: perceptions of faculty and resident physicians. J Am Med Inform Assoc 2004;11:300-9.

28. Carroll AE, Tarczy-Hornoch $P$, O'Reilly E, et al. Resident documentation discrepancies in a neonatal intensive care unit. Pediatrics 2003;111:976-80.

29. Carpenter I, Ram MB, Croft GP, et al. Medical records and record-keeping standards. Clin Med (Lond) 2007;7: 328-31.

30. Kuttler K. Physician critical care progress note: a study of the documentation of problems and plans. Utah, USA: University of Utah, 2008. 
31. Häeyrinen K, Saranto K, Nykäenen P. Definition, structure, content, use and impacts of electronic health records: a review of the research literature. Int J Med Inform 2008;77:291-304.

32. Zierler-Brown S, Brown TR, Chen D, et al. Clinical documentation for patient care: models, concepts, and liability considerations for pharmacists. Am J Health-Syst Ph 2007;64:1851-8.

33. Kibble J, Hansen PA, Nelson L. Use of modified SOAP notes and peer-led small-group discussions in a medical physiology course: addressing the hidden curriculum. Adv Physiol Ed 2006;30:230-6.

34. Donnelly WJ. Viewpoint: patient-centered medical care requires a patient-centered medical record. Acad Med 2005;80:33-8.

35. Grogan E, Speroff T, Deppen S, et al. Improving documentation of patient acuity level using a progress note template. JACS 2004;199:468-75.
36. Buchner D, Bagshaw S, Dodek P, et al. Prospective cohort study protocol to describe the transfer of patients from intensive care units to hospital wards. BMJ Open 2015;5:1-7.

37. Etchells E, Darzins P, Siberfeld M, et al. Assessment of patient capacity to consent to treatment. J Gen Intern Med 1999;14:27-34.

38. Strauss A. Qualitative analysis for social scientists. Cambridge University Press, 1987.

39. Hesse-Biber S, Leavy P, Yaiser M. Feminist approaches to research as a process: Reconceptualizing epistemology, methodology, and method. In: Hesse-Biber S, Yaiser M, ed. Feminist perspectives on social research. New York, NY: Oxford University Press, 2004:3-26.

40. Gwet K. Handbook of inter-rater reliability: the definitive guide to measuring the extent of agreement among raters. 4th edn ed. Gaithersburg, MD: Advanced Analytics, LLC, 2014. 